\title{
Sciences auxiliaires de l'histoire médiévale
}

Monsieur Olivier Guyotjeannin, Monsieur Denis Muzerelle, Monsieur Robert

Favreau, Madame Françoise Dumas, Monsieur Michel Pastoureau, Madame Marianne Mulon

\section{Citer ce document / Cite this document :}

Guyotjeannin Olivier, Muzerelle Denis, Favreau Robert, Dumas Françoise, Pastoureau Michel, Mulon Marianne. Sciences auxiliaires de I'histoire médiévale. In: Actes des congrès de la Société des historiens médiévistes de l'enseignement supérieur public, $20^{\mathrm{e}}$ congrès, Paris, 1989. L'histoire médiévale en France. Bilan et perspectives. pp. 471499;

doi : https://doi.org/10.3406/shmes.1989.1522

https://www.persee.fr/doc/shmes_1261-9078_1991_act_20_1_1522

Fichier pdf généré le 01/04/2019 


\title{
Sciences auxiliaires de l'histoire médiévale
}

\author{
Olivier Guyotjeannin \\ avec la collaboration de \\ Denis Muzerelle, Robert Favreau, \\ Françoise Dumas, Michel Pastoureau, \\ Marianne Mulon*
}

Les diverses disciplines traditionnellement centrées sur le recensement, l'examen critique et la publication des sources, écrites ou figurées, de l'histoire ont mérité à ce travail leur vocable premier de "sciences auxiliaires ». La taxinomie est loin d'être claire, et, devant l'élargissement des sources, de nombreux « rameaux de sciences collaboratrices tendent à se constituer en véritables sciences auxiliaires de l'histoire ${ }^{1}$ ", tournant par exemple autour d'une géographie historique enfin élargie, ou de la démographie. Le présent rapport n'a pris en compte que les plus traditionnelles d'entre elles.

Pour anciennes qu'elles soient, pour divers que soient leurs objets - diversité qui nous contraindra à une présentation excessivement rapide - , un même mouvement de fond caractérise les disciplines ici évoquées. Indépendamment même de la poursuite, voire de la naissance, de corpus de sources livrées aux enquêtes historiques, les sciences dites auxiliaires ont en effet connu un profond ćlargissement des curiosités, un remarquable renouvellement des méthodes comme des ambitions : mouvement amorcé en pleine «ère du doute », lancé de fait dès les

* La partie I et générale est due à O. Guyotjeannin, II à D. Muzerelle, III à R. Favreau, IV à Fr. Dumas, V et VI à M. Pastoureau, VII à M. Mulon. 
années cinquante-soixante, mais dont les retombées sont manifestes dans les deux dernières décennies. Loin d'être seulement des « auxiliaires », ces disciplines ont fait de leurs objets d'étude des sources pour elles-mêmes, dont la structure, l'agencement, la perception même par les contemporains sont autant de révélateurs des sociétés et des cultures médiévales. Sans même parler du récent renouvellement de la métrologie ${ }^{2}$, l'on a vu ainsi l'étude des types monétaires ou de la diffusion des sceaux éclairer l'histoire du pouvoir; l'anthroponymie quantitative ou l'héraldique appuyer l'histoire de la société et des structures familiales; la codicologie ou l'épigraphie épauler l'histoire culturelle.

\section{SOURCES D'ARCHIVES ET DIPLOMATIQUE}

Le repérage et l'examen critique des sources d'archives sont revendiqués par une nouvelle discipline, très inégalement développée selon les secteurs, où confluent archivistique, histoire des institutions, diplomatique traditionnelle et étude comparative, et que l'on pourra appeler « typologie des sources », à l'instar de nos collègues belges. Elle est illustrée à l'étranger, sans rien d'analogue en France, par divers manuels ou collections, tels les volumes de R.C. Van Caeneghem et F.L. Ganshof (dont une traduction française est enfin en cours) ou G.R. Elton pour l'Angleterre, et la justement célèbre « Typologie des sources du Moyen Age occidental » dirigée par L. Génicot. En dehors même de quelques contributions régionales, la voie vers un nouveau type d'instrument de recherche a été ouverte sous la direction de R.-H. Bautier ${ }^{3}$.

La même tendance semble bien se faire jour dans les publications des archives, où l'accent a été mis délibérément sur la couverture complète, à haute altitude, des fonds. Les deux dernières décennies ont bien vu, aux Archives nationales, la poursuite de quelques grandes séries d'inventaires, registres du Trésor des chartes, Parlement civil et criminel, anciens « hommages de France » de la Chambre des comptes, sans même 
évoquer divers fonds privés (Saint-Fargeau, Rohan-Bouillon, Maison de France, Castries, Uzès, Léran); de même, dans les Archives départementales et communales, plusieurs répertoires de séries partiellement médiévales, ecclésiastiques en tête. Ici et là, la grande affaire a été la publication, à un rythme soutenu, de l'Etat général des fonds et de l'Etat des inventaires des Archives nationales, de l'Etat des inventaires des Archives départementales, communales et hospitalières, de nombreux guides, enfin, d'Archives départementales ${ }^{4}$.

Le volet du repérage archivistique sera clos par l'évocation d'entreprises assistées par l'informatique et détaillées à ce titre dans un autre rapport : cartulaires et actes originaux antérieurs à 1221, recensés, filmés et indexés par l'IRHT (Paris-Orléans); actes originaux antérieurs à 1121, par les soins de l'ARTEM (Nancy).

Vieille tradition au pays des Mauristes, la diplomatique française est bien plus volontiers pratique que théorique; l'on s'explique ainsi que la part maigre soit faite à la diplomatique générale, et que bien des efforts convergent dans les activités d'édition, où ont été enfin définies, dans un cadre international, des normes... qu'il reste à appliquer et à diffuser ${ }^{5}$.

D'une diplomatique « qualitative », influencée par l'école de Vienne et attentive à sa suite aux faits de culture, on peut citer quelques exemples ${ }^{6}$, le nouveau départ donné à l'étude de l'acte du haut Moyen Age (J. Vezin aux côtés de H. Atsma), une synthèse sous presse de R.-H. Bautier sur le thème "Chancellerie et culture ", les importantes contributions de Ch. Vulliez à l'étude de l'ars dictaminis, préalables à une étude quantitative du discours diplomatique, qui doit dépasser l'approche analytique. De même, dans le domaine de la chronologie, les études des styles de datation se doublent d'un intérêt nouveau pour la perception du temps (qui ressortit plutôt à l'anthropologie historique) et pour la mention, faite ou non, du prince dans la date (actes catalans, normands, époque des Robertiens). Et si le discrimen veri ac falsi semble toujours relever du particulier, une voie nouvelle - et ardue - a été ouverte par J. Dufour, avec une étude statistique, ventilée par règne et période de falsification, des faux attribués aux carolingiens «français " 7 . 
La diplomatique spéciale a connu un très inégal développement. Viennent en tête du palmarès, selon des critères variés, les actes royaux, les actes privés, les actes en langue vulgaire ${ }^{8}$. Les actes royaux ont vu, toujours par les soins de l'Académie des inscriptions, l'achèvement de la série des Carolingiens « français » et de Philippe Auguste (sauf index et additions), la poursuite du chantier pour les $\mathrm{XI}^{\mathrm{e}}-\mathrm{XII}^{\mathrm{e}}$ siècles (Louis VI sous presse) et Philippe le Bel : ici, comme en bien d'autres domaines, le $\mathrm{XIII}^{\mathrm{e}}$ siècle demeure déshérité. En dehors de la synthèse de R.-H. Bautier sur les Carolingiens, c'est le bas Moyen Age qui s'est attiré les études les plus fournies, dans des directions traditionnelles : mentions hors-teneur, chancellerie et prosopographie de son personnel (A. Lapeyre et R. Scheurer); d'autres secteurs, tels les formulaires, sont largement en friches, sauf à signaler un projet animé par $\mathrm{Ph}$. Contamine et Fr. Autrand. Même richesse, non plus des éditions, mais des études, dans le domaine de l'acte privé, dont la diversité française a attiré bien des chercheurs ${ }^{9}$. Quelques études aussi sur la question du recours à la langue vulgaire, mais surtout, en ce domaine, une vigoureuse impulsion donnée à l'édition du corpus des Documents linguistiques de la France, dirigé par J. Monfrin.

Les actes pontificaux ont vu la poursuite des publications de l'Ecole française de Rome (Jean XXII, Urbain V, Innocent VI) et un début de participation française à d'autres entreprises internationales ${ }^{10}$; les études se font ici plus rares, le personnel de la chancellerie ayant fait l'objet de plusieurs articles de B. Barbiche. Le bilan est encore plus mélangé avec les actes princiers, où n'ont guère été publiés que des prééditions lorraines et un catalogue pour l'Anjou du $\mathrm{xI}^{\mathrm{e}}$ siècle (O. Guillot), d'autres éditions demeurant dactylographiées (comtes de Périgord, Eudes IV de Bourgogne, Humbert II de Viennois; premiers registres des chancelleries de Bretagne et d'Armagnac - ces derniers à paraître); de rares études sur la Bourgogne et le Dauphiné ont été, la plupart du temps, éditées à l'étranger. Il faut donc souhaiter que l'Académie des inscriptions puisse rapidement ouvrir sa nouvelle série d'《 Actes princiers ", où l'on attend déjà les actes bretons antérieurs à 1148 et ceux de BloisChampagne jusqu'à 1284. L'on doit de même attendre les fruits 
du travail du Groupement de recherche animé par M. Parisse, pour voir l'édition des actes épiscopaux sortir de son quasi-néant actuel : Reims, Metz, Toul et Poitiers devraient en profiter les premiers.

Reste, difficile à classer, le domaine des recueils ou éditions thématiques au sens large, où se distinguent quelques lueurs : belles mais rares éditions d'inventaires après décès; recueils régionaux, de loin dominés par l'achèvement des Chartes $d u$ Forez jusqu'à 1300 ; vogue, méritée, des chartes de franchises et de coutumes, dont se détachent trois recueils systématiques consacrés à la Picardie, à la Savoie et à l'Agenais.

Si l'on considère les documents en fonction des fonds où ils se trouvent, ce sont, sans surprise, les fonds ecclésiastiques qui se taillent la part du lion. Tout d'abord par quelques beaux instruments d'investigation : la fin du «Cottineau », la poursuite des Abbayes et prieurés de l'ancienne France, auxquels dom Becquet fournit à peu près tous les cinq ans un nouveau volume tiré de la Revue Mabillon (diocèses actuels d'Arras, Amiens, Soissons, Beauvais), alors que la Gallia Monastica, sans doute trop ambitieuse, n'a pas dépassé le premier volume (diocèse de Reims) ${ }^{11}$. Les éditions de cartulaires et chartriers semblent connaître, sans vouloir être trop optimiste, comme un début de reprise après une période très critique. Sans même mentionner les contributions partielles ou préparatoires ${ }^{12}$, de très beaux ensembles ont été livrés aux historiens : Saint-Georges de Hesdin et Fontenelle, Remiremont et Bouxières, Sainte-Chapelle de Vincennes et, pour deux volumes, Saint-Magloire de Paris, Saint-Vincent du Mans, Pontigny, Montierneuf de Poitiers, Fontaine-le-Comte et Saint-Amand de Boixe, Obazine, Vabres, Lézat et La Selve, Bonnefont, chapitre d'Agde, Trinquetaille et Montrieux : il est vraisemblable qu'après la grande vague des études d'histoire régionale, pour lesquelles le chercheur parvient à dominer seul la documentation, le nouveau départ des enquêtes « transversales », recourant fréquemment à des études de vocabulaire, rend plus pressant le besoin d'éditions comme de corpus informatisés. Les projets d'éditions sont nombreux et importants (La Sauve-Majeure, La Grasse, Fontevraud, etc.). Il reste que les possibilités financières de l'édition 
nationale ne suivent pas : Corbie, La Grasse et Saint-Nicaise de Reims à part, rien ne semble prévu pour publier des éditions dactylographiées, comme celles des cartulaires de Saint-Serge d'Angers, Notre-Dame d'Evron, Levroux, Moissac, pour ne citer que quelques noms.

Les autres documents des fonds ecclésiastiques subissent la logique du tout ou rien : certaines catégories disposent de séries, bien programmées, de répertoires, synthèses, éditions, tels les documents nécrologiques (J.-L. Lemaître et quelques autres éditeurs), les visites pastorales (N. Coulet et quatre volumes de répertoire collectif), les statuts synodaux (O. Pontal et J. Avril), les pouillés (pour lesquels la province de Bordeaux doit venir clore la série lancée par l'Académie des inscriptions). Cluny peut encore avancer une belle collection de Statuts, chapitres généraux et visites; mais pour le reste, registres d'inquisition, finances pontificales, enquêtes, une pénurie accablante, où l'on ne peut guère avancer à chaque fois qu'une ou deux éditions. Le bilan est encore plus désolant dans le domaine des comptabilités.

Les archives laïques ont moins de succès. Quelques éditions sont éclipsées par deux beaux ensembles méridionaux, maison de Durfort (N. de Peña) et début du trésor des chartes d'Albret (J.$B$. Marquette). Editions importantes mais guère moins isolées de documents tirés des archives municipales : les documents fiscaux de Saint-Flour et de Paris, le registre des compagnies françaises de Paris, les comptes de Montréal, les actes de Péronne ne peuvent faire oublier l'inachèvement du Répertoire des délibérations et comptabilités communales. Seul point positif, les éditeurs français sont encore épargnés par les cas de conscience que posent à leurs collègues belges les mérites respectifs de l'édition intégrale, du tableau récapitulatif et du microfilmage !

Les documents de gestion domaniale bénéficient aujourd'hui d'une synthèse de $R$. Fossier et d'éditions, dont le plus grave défaut est moins le faible nombre - encore que je n'en aie recensé qu'une quinzaine - que l'absence totale de coordination dans leur publication. De très beaux textes ont reçu des éditions exemplaires, tels les Documents comptables de Saint-Martin de Tours (P. Gasnault); les Enquêtes sur les droits et revenus de Charles $I^{\text {er }}$ en Provence (E. Baratier); des censiers du XIII ${ }^{\mathrm{e}}$ siècle, 
inventaires méthodiques des profits seigneuriaux, ainsi pour l'évêché de Toul (H. Olland) et Saint-Pierre-aux-Monts de Châlons (A. Saint-Denis); ou ce démarquage de cartulairechronique, composé au début du XII ${ }^{\mathrm{e}}$ siècle à Marchiennes (B. Delmaire). La situation devient lamentable pour les comptabilités seigneuriales, où les « raisons » de Guillaume de Murol (P. Charbonnier) tranchent par leur volume et leur intérêt, comme pour les documents féodaux, où se signale le Livre d'hommages de Gaston Phébus ( $P$. Tucoo-Chala), dont la position périphérique a autorisé une publication à Saragosse!

Les archives des administrations, royale et princières, ont, elles aussi, livré des éditions importantes mais presque aussi isolées : un beau dossier d'ambassadeur en 1400-1403 (I. Le Bis), les registres du conseil ducal de Bretagne en 1459-1463 (A. Lejeune, dact.), les documents du Clos des Galées de Rouen (A. Merlin-Chazelas); quelques beaux documents fiscaux, dont une enquête de la Flandre wallonne (A. Derville), des rôles artésiens (P. Bougard et M. Gysseling) et normands (M. Nortier). De même peut-on signaler, pour les documents financiers, par les soins de l'Académie des inscriptions et belles-lettres, l'achèvement des Comptes généraux de l'Etat bourguignon (1416-1420) (M. Mollat et R. Favreau), le premier volume du Catalogue des comptes royaux (1328-1364), dû au regretté $\mathrm{R}$. Cazelles, le chantier des comptes sur tablettes de cire de l'Hôtel du roi au $\mathrm{XIII}^{\mathrm{e}}$ siècle (E. Lalou); grâce à la Commission royale belge, l'édition des comptes du receveur d'Artois pour 1303-1304 (B. Delmaire).

En résumé, une diplomatique aussi pratique que peu spéculative, qui nous a fourni quelques synthèses, ouvert des voies stimulantes, rarement ennuyé - ce qui est déjà un acquis -, mais garde encore bien de l'ouvrage et appelle la refonte des manuels classiques; une typologie qui doit encore faire ses preuves ; des éditions qui ont survécu à la tourmente et connaissent peut-être un léger mieux, pour lesquelles se confirme le rôle primordial d'institutions centrales (Académie des inscriptions, CNRS, CTHS), où il faut encore attendre pour juger des effets de la décentralisation, qui remédiera, espérons-le, à la crise des 


\section{L'HISTOIRE MÉDIÉVALE EN FRANCE}

sociétés savantes locales, et surtout où les efforts demeurent extrêmement (excessivement ?) dispersés et individualistes.

\section{Paléographie et codicologie}

On n'abordera ici - sauf exception - que le domaine des écritures latines médiévales ${ }^{13}$. Mais c'est précisément par une exception qu'il convient de commencer, pour rappeler les travaux de J. Mallon sur l'écriture romaine.

En effet, si l'objet spécifique de ses recherches échappe au cadre de la médiévistique, les principes évolutionnistes énoncés dès 1953 ont largement bouleversé la paléographie médiévale, en lui offrant de nouvelles méthodes et de nouveaux fondements. C'est dans un film, Ductus ${ }^{14}$, qu'on doit voir l'aboutissement de son œuvre : le dessin animé scientifique s'y révèle le meilleur moyen de rendre compte simultanément du dynamisme du geste graphique et de celui de l'évolution diachronique des tracés. Le recueil de ses principaux articles ${ }^{15}$, assorti de nombreux commentaires et mises à jour (voire rétractations), laissait attendre une refonte de son maitre traité; la mort ne la lui laissa pas entreprendre.

Pour la paléographie médiévale proprement dite, il est d'usage de distinguer le domaine des écritures documentaires de celui des écritures livresques. Cette dichotomie assez académique, inégalement justifiée selon les périodes, reflète moins une différence de matériau ou de méthode que le rôle dévolu à l'analyse des écritures selon les domaines : simple élément de critique dans un cas, indispensable technique de datation et de localisation dans l'autre. C'est probablement cette moindre motivation qui explique la relative désaffection pour les écritures documentaires qui s'est toujours manifestée chez les médiévistes français et ne s'est guère démentie au cours des dernières décennies.

Les périodes anciennes suscitent cependant davantage d'intérêt. Pour ces dernières années, le principal apport consiste dans la publication des Chartae latinae antiquiores, sous la direction de 


\section{SCIENCES AUXILIAIRES}

R. Marichal. Les fac-similés antérieurs de diplômes mérovingiens et carolingiens se trouvent largement supplantés par les volumes concernant la France ${ }^{16}$, et la prise en compte de documents ecclésiastiques et privés, ou non diplomatiques (telles les authentiques de reliques du vIII ${ }^{\mathrm{e}}$ siècle découvertes à Chelles en 1983), élargit notablement la documentation. On attend l'étude paléographique d'ensemble que $J$. Vezin doit prochainement publier dans un volume d'introduction. Un avant-goût de ce qu'elle pourra apporter nous a été fourni par l'analyse fouillée qu'il a donnée des comptes de Saint-Martin de Tours ${ }^{17}$, spécimens exceptionnels d'écriture usuelle au viI ${ }^{\mathfrak{e}}$ siècle.

Passé l'époque carolingienne, les travaux se font rarissimes et l'on ne peut guère citer que les recherches de F. Gasparri sur les écritures de la chancellerie royale au $\mathrm{XII}^{\mathrm{e}}$ siècle ${ }^{18}$. Ses conclusions ont connu quelques critiques; mais elles jettent un pont important entre les deux principaux aspects de la paléographie médiévale en révélant le rôle des scriptoria des grandes abbayes parisiennes dans la confection des actes. Quelques travaux plus récents ${ }^{19}$, mais encore dispersés, laissent peut-être entrevoir un regain d'intérêt pour l'étude des écritures diplomatiques; l'influence de l'école belge de J. Stiennon y est notable.

C'est encore en matière de paléographie documentaire que la demande en matériau didactique est la plus vive. Un excellent recueil d'initiation aux écritures cursives les plus difficiles nous a été procuré par E. Poulle ${ }^{20}$; sa récente édition des cédules de l'assemblée de $1398^{21}$ fournit un bel échantillonnage d'écritures personnelles. Pour le reste, les archivistes départementaux, à qui incombe le plus souvent le soin d'initier le public au déchiffrement, tentent en ordre dispersé de pallier le manque d'instruments par des publications d'intérêt local - avec plus ou moins de bonheur 22 ! C'est peut-être ici le lieu de suggérer qu'ils se concertent pour définir les besoins de leur public et élaborer en commun un outil solide et efficace.

Le domaine de la paléographie livresque est beaucoup plus florissant, l'essor actuel correspondant souvent au développement d'initiatives lancées dès les années cinquante, notamment à la suite de la création du Comité international de paléographie 


\section{L'HISTOIRE MÉDIÉVALE EN FRANCE}

(latine) (CIPL). Principale entreprise alors mise en chantier, le "Catalogue des manuscrits datés » (CMD) continue de paraître, pour la France à un rythme soutenu, depuis 1959 . Les sept tomes parus ${ }^{23}$ couvrent maintenant la majeure partie de nos fonds (à l'exception seulement du Nord de la France et du Fonds français de la Bibliothèque nationale). Ils ont non seulement fourni aux paléographes d'abondants spécimens datés d'écritures variées (environ 3000 clichés), mais, en accordant plus d'importance que d'autres collections analogues à l'origine géographique des volumes, ils ont jeté un jour nouveau sur les scriptoria et bibliothèques du Nord-Est (Reims, Saint-Thierry, Verdun), de Bourgogne (Cîteaux, Clairvaux, Cluny), de Normandie et des pays de Loire (Mont-Saint-Michel, Saint-Wandrille, Fécamp; Orléans, Angers, Vendôme), voire pratiquement mis au jour des ressources inattendues (fonds de Belval et de Signy à la bibliothèque de Charleville).

L'importance de la collection peut encore se juger aux publications auxquelles elle a servi de modèle. Outre les équivalents nationaux dans la plupart des pays d'Europe, en France même ont été élaborés selon une formule analogue le Catalogue des manuscrits contenant des notations musicales (par S. Corbin et M. Bernard, 1964-1972, 3 vol.) et le Catalogue des manuscrits datés en caractères hébraïques (1972-1981, 3 vol., en collaboration avec les institutions israéliennes). Peut-on pour autant estimer que cette manne d'échantillons compense la carence totale de notre pays en matière de publication de manuscrits en fac-similé?

Parmi les autres travaux, les études de scriptoria occupent la place d'honneur. Certaines sont plus ou moins étroitement liées aux recensements effectués dans le cadre du CMD. Pour traditionnel que soit le genre, on peut y déceler quelques tendances nouvelles : d'une part, une attention plus soutenue et parfois neuve - aux éléments " péri-graphiques » et codicologiques (abréviations, ponctuation...; réglure, mise en page, reliure...) ${ }^{24}$; d'autre part, le souci de confronter la production livresque aux actes émanés du même établissement ${ }^{25}$.

Un autre domaine de prédilection des paléographes est celui des « autographes » médiévaux (manuscrits d'auteur ou de 
travail, scriptoria de savants ou de littérateurs). De fort nombreux articles ont abordé ce sujet depuis une vingtaine d'années, laissant apparaître, là encore, une évolution des préoccupations. L'accent y est désormais moins mis sur la biographie du personnage et la critique textuelle que sur l'analyse des méthodes de travail intellectuel ${ }^{26}$.

Mais ce qui a peut-être le plus profondément marqué la paléographie (en France comme ailleurs) au cours de ces dernières années a été l'émergence à ses côtés de la codicologie - dont le nom n'aurait peut-être pas même été mentionné dans un rapport analogue, il y a vingt ou trente ans ou alors dans une acception sensiblement différente (cf. L'Histoire et ses méthodes, 1961). Dans ce nouveau cadre méthodologique, on peut distinguer trois courants, ou peut-être trois niveaux correspondant à des implications croissantes dans le domaine historique.

Tout d'abord, une codicologie analytique et descriptive, qui s'efforce de reconstituer exactement l'ensemble des techniques de fabrication du livre médiéval (on a signalé plus haut l'incidence de cette approche dans les travaux paléographiques). Là encore, l'élaboration d'instruments de travail est due à l'initiative du CIPL qui, dès les années soixante, recommandait l'établissement d'un glossaire technique international, dans un souci de clarification et de normalisation peut-être plus que de simple intercompréhension. La base de cet instrument a vu le jour en français ${ }^{27}$ et des adaptations nationales, devant mener au répertoire polyglotte, sont en cours dans plusieurs pays.

L'introduction des techniques de laboratoire ${ }^{28}$, notamment dans le cadre du Centre de recherche sur la conservation des documents graphiques (créé par le CNRS en 1963), a ouvert de nouveaux horizons. En France, l'effort a principalement porté, ces derniers temps, sur l'identification et l'histoire des encres ${ }^{29}$ et des pigments. Les papiers médiévaux (occidental et oriental) ont fait eux aussi l'objet de recherches importantes et novatrices ${ }^{30}$.

Sur un autre niveau viennent se placer les recherches visant à la reconstitution des fonds de bibliothèques médiévales, sur la base d'inventaires anciens et de documents d'archives. Si l'orientation n'est pas véritablement neuve, elle a reçu une très 
vigoureuse impulsion depuis un quart de siècle grâce aux travaux d'A. Vernet ${ }^{31}$. L'énorme documentation relative aux bibliothèques monastiques, qui a été rassemblée par ses soins ou sous sa direction à l'Institut de recherche et d'histoire des textes, constitue en elle-même un acquis capital, et cet effort de recensement des sources vient de voir son aboutissement dans la publication d'un répertoire national des inventaires de bibliothèques $^{32}$.

Après une période où ont fleuri les monographies de petites bibliothèques, monastiques ou privées, la recherche est maintenant en mesure d'affronter des fonds plus importants, en volume et en rayonnement. Ceux de Clairvaux ${ }^{33}$, de Saint-Victor ${ }^{34}$, de Saint-Denis ${ }^{35}$ ont été minutieusement reconstitués et analysés ; d'autres établissements cisterciens de premier plan (Cîteaux, Pontigny...) font actuellement l'objet de travaux. Il faut y ajouter, dans un domaine un peu différent, les recherches consacrées aux bibliothèques d'ecclésiastiques, sous la papauté d'Avignon, à partir des archives du droit de dépouille ${ }^{36}$.

Une troisième orientation s'emploie enfin à interpréter les particularités archéologiques du manuscrit et de l'incunable en fonction de critères économiques et sociaux autant qu'intellectuels ou technologiques. Etudiant les caractéristiques de vastes ensembles de livres et recourant systématiquement à la statistique pour interpréter les observations, cette « codicologie quantitative " ambitionne d'aboutir à une histoire motivée du livre médiéval, envisagé comme le reflet du contexte humain qui l'a produit.

Dans ce domaine, où la France joue véritablement un rôle pionnier, la recherche est encore en pleine phase expérimentale et la méthodologie se forge au fil des articles et communications. On n'en est donc pas encore à pouvoir renvoyer à un travail de synthèse ; mais les fondements de la démarche ont été solidement cxposés ${ }^{37}$ et son efficacité a été illustrée de façon convaincante, en particulier dans l'étude de la production manuscrite au cours du Moyen Age ${ }^{38}$. D'autres jalons importants ont été fixés sur d'autres points (notamment ceux qui ont trait à la lisibilité de la page et du texte) par les publications du groupe «Quanticod ${ }^{39}$ - car c'est une autre caractéristique de cette métho- 
dologie que d'exiger un travail collectif, là où la recherche individuelle était de règle jusqu'à présent.

C'est donc, à l'évidence, dans le domaine de la codicologie que la recherche a manifesté le plus de vigueur et qu'on a bénéficié des apports les plus consistants, depuis dix ans tout particulièrement. Cela ne doit cependant pas prêter à confusion, car les deux disciplines restent intimement liées, et reposent bien souvent sur les mêmes chercheurs. Et si la codicologie tente de plus en plus ouvertement de se faire reconnaître comme une discipline à part entière (statut qu'elle a déjà obtenu dans plusieurs pays), c'est moins pour s'affranchir de la paléographie que pour lui ouvrir un horizon renouvelé.

\section{EPIGRAPHIE}

Erudits et amateurs d'histoire se sont de tout temps intéressés aux inscriptions que le médiéviste ne cesse de rencontrer, aussi bien dans les églises romanes ou gothiques que dans les textes. Au cours des vingt dernières années, il est aisé de repérer, grâce à la Bibliographie annuelle de l'histoire de France, bon nombre de contributions qui signalent de nouvelles inscriptions ou apportent des compléments sur des épigraphes déjà connues. Le plus souvent, il s'agit d'articles très brefs, dans des revues locales à diffusion restreinte. Ces études sont toujours utiles en raison de la connaissance du terrain qu'ont leurs auteurs, et elles aident à situer un matériel épigraphique très dispersé. Elles intéressent généralement une seule inscription. Plus rarement, elles concernent un monument, une ville, une région, par exemple les inscriptions funéraires et campanaires de la cathédrale de Rouen, les pierres tombales de la cathédrale de Meaux, les inscriptions de l'abbaye de Longpont ou de l'abbatiale NotreDame de Mouzon, les vingt-cinq épitaphes carolingiennes de Saint-Outrille-du-Château à Bourges, ou encore les inscriptions romanes d'Alsace, les inscriptions du département du Tarn ou de 
l'Indre. Parfois elles se regroupent autour d'un thème : les chrismes du Sud-Ouest, les très controversés graffiti templiers de Chinon, ou d'une catégorie particulière, telles les runes étudiées à différentes reprises par L. Musset.

On mettra à part la publication d'inscriptions transmises par les épitaphiers ou par les relevés et dessins de Gaignières (J. Adémar, G. Dordor, J.-B. de Vaivre). L'apport des chroniqueurs peut être d'une très grande importance, tel un André de Fleury (R.-H. Bautier). L'épigraphiste doit encore la plus grande attention aux catalogues d'expositions, inventaires de musées, corpus concernant le Moyen Age, tel celui des émaux médiévaux par M.-M. Gauthier, ou des vitraux, en particulier la grande étude de L. Grodecki sur les vitraux de Saint-Denis.

La publication, en 1986, par G. Nahon des Inscriptions hébraïques et juives de la France médiévale a été le point d'aboutissement d'un projet conçu en 1973 par B. Blumenkranz, ainsi que de rapports de mission dans le Sud-Ouest et en Lorraine et Alsace, d'études sur les inscriptions juives d'Arles, de Mantes-la-Jolie, de Dijon, d'une syntèse sur l'épigraphie juive méridionale ${ }^{40}$, le tout publié entre 1968 et 1980.

La nouveauté la plus marquante de ces vingt dernières années pour l'épigraphie médiévale en France reste la recension générale et la publication systématique des inscriptions des $\mathrm{VIII}^{\mathrm{e}}$ $\mathrm{XIII}^{\mathrm{e}}$ siècles. C'est en 1969 qu'une formation du CNRS a été créée pour dresser un Corpus des inscriptions de la France médiévale. Installée à Poitiers auprès du Centre d'études supérieures de civilisation médiévale, cette équipe établit un fichier général des inscriptions - incluant aussi le $\mathrm{XIV}^{\mathrm{e}}$ et le $\mathrm{XV}^{\mathrm{e}}$ siècle - étudie sur place tous les textes des VIII $^{\mathrm{e}}$-XIII ${ }^{\mathrm{e}}$ siècles, et publie les résultats de l'enquête au rythme d'un volume de 250 à 300 pages chaque année depuis 1974. A ce jour l'enquête sur place couvre 48 départements, et ont été publiées en 14 volumes près de 2000 inscriptions de 29 départements.

La recension assurée par l'équipe de Poitiers est menée de pair avec l'établissement d'instruments de recherche propres à la discipline, l'exploration et l'exploitation des différents champs auxquels l'épigraphie peut apporter une contribution. R. Favreau a publié en 1979 la première synthèse sur les inscriptions 
médiévales ${ }^{41}$ et a présenté plusieurs études générales sur la discipline ${ }^{42}$. Le fichier épigraphique général établi à Poitiers a été traité par l'informatique, et les concordances, par leur exhaustivité et l'apport du contexte de chaque mot, permettent de repérer formules, expressions, emprunts. Ce fichier sera transformé en banque de données en 1989-1990. Une publication d'ensemble sur le formulaire des inscriptions médiévales en France est en chantier, et plusieurs études particulières ont été publiées en ce domaine par R. Favreau. Des recherches sont menées systématiquement sur les sources bibliques, liturgiques, hagiographiques, patristiques, littéraires. On retiendra en particulier une thèse sur les Inscriptions de consécration d'autels et de dédicace d'églises en France du VIII ${ }^{e}$ au XIII siècle : épigraphie et liturgie (J. Michaud), une étude des inscriptions de l'église de Saint-Savin-sur-Gartempe - où R. Favreau montre notamment les correspondances entre les inscriptions de consécration des autels du déambulatoire et les peintures murales de la crypte une autre thèse sur les inscriptions des prophètes en Occident (F. Gay). L'étude des sources, auteurs païens et chrétiens, peut conduire aussi bien aux Institutes de Justinien qu'à Colius Sedulius, à Baudri de Bourgueil ou à bien d'autres, le champ de recherche restant largement ouvert et à peine abordé ${ }^{43}$.

Le domaine de l'écriture des inscriptions n'a été que fort peu traité en France depuis l'article fondamental publié par P. Deschamps dans le Bulletin monumental en 1929. A Poitiers, la mise sur fiches systématique de toutes les lettres des inscriptions photographiées de la France du Sud pourrait déjà permettre, pour ce champ géographique, des travaux solidement étayés. Les larges perspectives de recherche offertes par la langue des inscriptions, évolution du latin médiéval, apparition de la langue vulgaire, niveaux de culture, étude du vocabulaire, etc., n'ont été encore que fort peu exploitées.

Même si les travaux des vingt dernières années y placent la France en très bonne place, les perspectives qui s'ouvrent à ce domaine encore neuf demeurent donc considérables. 


\section{Numismatique}

La numismatique tient sa spécificité de l'étude des monnaies et des médailles. Elle s'appuie aussi sur les documents écrits et sur les données archéologiques dont elle peut contribuer à compléter ou à confirmer l'enseignement.

Aucun plan d'ensemble n'a suscité un développement coordonné des études, qui sont conduites par des individus plus que par des équipes.

Cette situation a des effets positifs : tout d'abord une grande disponibilité devant les découvertes fortuites et les trouvailles faites au cours des fouilles archéologiques. La multiplication de ces dernières, leur minutie, leur orientation vers des sites peu spectaculaires font que l'accent est mis désormais sur le commun, l'usuel, souvent le plus usé et le plus difficile à identifier. En conséquence, on constate une certaine évolution dans l'étude de la monnaie qui porte plus souvent sur l'obole du pauvre que sur l'écu du riche. Autre effet positif, une certaine souplesse pour accueillir et exploiter les progrès des sciences exactes, en l'occurrence l'analyse métallique par méthodes physiques non destructives qui permet, surtout en l'absence de textes, de connaître les constituants de la monnaie, son titre au premier chef.

Soulignons l'aspect négatif de cette situation : pas de traité, pas de manuel dignes de ce nom, seulement un remake du catalogue de J. Lafaurie consacré aux monnaies royales capétiennes, allégé et complété par J. Duplessy ${ }^{44}$. Sachons enfin que ce domaine n'est pas chasse gardée ${ }^{45}$ : les numismates anglais, belges, hollandais s'intéressent à la Flandre, à la Guyenne. Un Américain, T. Bisson, a analysé de rcmarquable manière les mouvements populaires qui tendaient à imposer une stabilité de la monnaie aux $\mathrm{XI}^{\mathrm{e}}$ et $\mathrm{XII}^{\mathrm{e}}$ siècles.

Il est classique, en numismatique française, de distinguer la période du denier de celle du gros. Le denier, pièce d'argent créée à la fin du viI ${ }^{\mathrm{e}}$ siècle, demeure la seule monnaie employée 
jusque pendant le règne de Louis IX. A partir de 1266, les monnaies de référence sont le gros de douze deniers ${ }^{46}$ et les monnaies d'or.

Pour la période du denier, J. Lafaurie a tracé un très bon panorama de la monnaie royale du haut Moyen Age qui peut servir d'introduction à cette première période. La partie carolingienne doit être revue à la lumière des analyses métalliques ${ }^{47}$.

$\mathrm{Au}$ titre des généralités, indiquons le répertoire des trésors monétaires trouvés en France, enfouis depuis l'époque carolingienne jusqu'à 1180 . J. Duplessy y a travaillé pendant plusieurs décennies sous la direction de J. Lafaurie, puis seul. C'est un utile instrument de travail ${ }^{48}$.

Si le monnayage de Raoul, roi de France au début du $x^{\mathrm{e}}$ siècle, est mieux connu grâce à plusicurs trouvailles récentes ${ }^{49}$, c'est surtout la fin de l'époque carolingienne qui a bénéficié d'une découverte exceptionnelle. Le trésor de plusieurs milliers de pièces mis au jour à Fécamp a permis de brosser un tableau de l'ensemble du monnayage de Francie occidentale à la fin de l'époque carolingienne ; l'examen des poids et de quelques titres fait apparaître l'évolution de la monnaie, les affaiblissements, les redressements qu'elle subit, sa diversité suivant les régions, en un mot sa complexité. L'examen des types et des légendes, de leur éloignement progressif ou brutal du modèle royal fournit une contribution importante à la connaissance des manifestations d'indépendance, parfois précoces, qui sont le fait de quelques monastères, de magnats laïcs, d'évêques enfin ( $F$. Dumas).

Le colloque consacré à Philippe Auguste a été l'occasion d'un autre tableau d'ensemble du monnayage dans le royaume, très divers et éparpillé. Les analyses effectuées montrent néanmoins que se dessinent des zones de circulation relativement ordonnées où certains deniers font office de doubles vis-à-vis d'autres qui sont toujours considérés comme l'unité ${ }^{50}$.

Parmi les études régionales, celles des monnaies normandes des $\mathrm{X}^{\mathrm{e}}$-XII ${ }^{\mathrm{e}}$ siècles a attiré l'attention sur ces documents extrêmement frustes dont la dispersion en France et en Europe reflète assez exactement les voies suivies par les Normands dans leurs pèlerinages et leurs conquêtes, à ceci près que l'on ne trouve plus de deniers normands outre-Manche après $1066^{51}$. Parallèlement, 
l'emploi des monnaies en Normandie d'après les textes a été étudié par L. Musset ${ }^{52}$.

La récente publication d'une monographie consacrée au monnayage des ducs de Bourgogne du $\mathrm{XI}^{\mathrm{e}}$ au Xv $v^{\mathrm{e}}$ siècle, portant sur le monnayage d'une seigneurie depuis son origine jusqu'à son extinction, fournit une transition vers la période du gros. On rencontre dans cet ouvrage tous les aspects évoqués par d'autres auteurs de façon plus partielle pour d'autres seigneuries : étude de la politique monétaire et de ses profits, des émissions, du numéraire et de la circulation, des procédures administratives, du personnel. On assiste en outre au lent épanouissement du pouvoir ducal capétien, à l'extension de la puissance des Valois finalement mise en échec par l'administration royale en dépit de la faiblesse du souverain ${ }^{53}$.

$\mathrm{Au} \mathrm{XIV}$ siècle, époque retenue par la plupart des savants ${ }^{54}$, les fabrications étaient intermittentes mais souvent très importantes (38 millions de pièces à Dijon en 1303, 4700000 pièces blanches et noires à Limoges en 1365-1366); les bénéfices en général élevés (14\% en Guyenne), parfois nuls (atelier pontifical de Pont-de-Sorgues) ; les assignations pour paiements rapides, remboursements ou garantie d'emprunts (Dauphiné, Guyenne) fréquentes; les concessions de frappes mal surveillées (Blois).

La remarquable étude de P. Capra sur la Guyenne anglogasconne se veut entièrement textuelle, laissant à un « collectionneur » le soin de mettre en accord les documents avec les monnaies retrouvées, dont le catalogue date d'un demi-siècle.

Des diverses études de M. Bompaire, fouillées et originales, nous retenons ici l'analyse pénétrante d'un livre de changeur du $\mathrm{XIV}^{\mathrm{e}}$ siècle, occasion d'un tableau des émissions à cette époque ${ }^{55}$.

Indiquons, pour terminer, que ces quelques lignes simplificatrices ne prétendent en aucune façon refléter le foisonnement des publications dans ce domaine mais visent à présenter celles qui semblent pouvoir rendre des services plus immédiats aux historiens. 


\section{Héraldique}

Les deux dernières décennies ont été pour les études héraldiques l'occasion de profondes mutations, pour ne pas dire d'une véritable révolution épistémologique ${ }^{56}$. Dans la mise en place de celle-ci, la part des chercheurs français (M. Pastoureau, M. Popoff, H. Pinoteau, P. Bony, Ch. de Mérindol, J.-B. de Vaivre et quelques autres) a été prépondérante. Profitant de l'éclatement des barrières au sein des siences sociales, puis de l'apport de l'anthropologie et de la sémiologie à l'histoire, ces chercheurs sont parvenus à faire des armoiries un objet historique à part entière et non plus seulement une marque de possession ou de commande, dont l'identification pouvait seulement être utile à l'archéologie ou à l'histoire de l'art. Les études nouvelles se sont ainsi développées autour de quatre axes :

1. Tout d'abord celui des rapports entre l'héraldique et la parenté : l'armoirie est avant toute chose un emblème familial et obéit à des lois de port et de transmission qui varient dans le temps et dans l'espace; elle participe en outre du patrimoine et de la mythologie emblématiques de chaque groupe familial, l'écu armorié renvoyant à la parenté étroite et le cimier qui le surmonte renvoyant à la parenté large.

2. Ensuite l'héraldique statistique et l'étude des phénomènes de fréquence ou de rareté des figures et des couleurs dans les armoiries d'une époque, d'une région, d'une classe ou catégorie sociale. C'est sur ces terrains que l'héraldique médiévale, en dressant cartes, tableaux, graphiques de toutes sortes, a le plus profondément transformé ses enquêtes, renouvelé ses méthodes et étendu ses investigations.

3. Puis l'examen du blason en tant que système de signes rigoureux et performant. Avec peu de données, ce système et la langue qui s'y rattache livrent une foule d'informations et permettent de passer sans difficulté aucune, à six ou huit siècles de distance, du texte à l'image et de l'image au texte, l'armoirie étant les deux à la fois. Parmi tous les langages documentaires 


\section{L'HISTOIRE MÉDIÉVALE EN FRANCE}

auxquels sont confrontés les historiens, c'est là un cas unique. Il faudrait qu'à présent, sur ces terrains, les linguistes et les sémiologues prennent le relais des héraldistes et des philologues du blason.

4. Enfin l'étude des armoiries et emblèmes attribués à des personnages imaginaires (héros de romans, créatures mythologiques, figures bibliques, saints et personnes divines, vices et vertus personnifiés, etc.). De telles enquêtes, mettant en relation ce que l'on sait ou ce que l'on croit du personnage avec les figures et les couleurs qui lui sont attribuées, permettent de cerner au plus près les procédés de symbolisation et d'emblématisation de la culture médiévale.

Ces approches renouvelées d'une science ancienne ont également permis de repenser le problème des sources et de mettre en chantier une nouvelle et fructueuse politique de publication de documents inédits, armoriaux et sceaux principalement. En ces domaines, rien n'aurait pu se faire sans la généreuse compréhension de plusieurs institutions (les Archives de France, le CNRS) et de quelques éditeurs privés (Editions du Léopard d'or, Editions Picard, Editions Christian). Souhaitons que cette politique se poursuive, malgré les coûts de plus en plus élevés que demandent les publications héraldiques, toujours grandes consommatrices d'images.

\section{Sigillographie}

Sans subir une transformation aussi radicale que l'héraldique, la sigillographie a connu elle aussi un essor remarquable au cours des vingt dernières années, et ici encore la part des chercheurs français a été dominante (R.-H. Bautier, Y. Metman, B. Bedos, M. Dalas-Garrigues, M. Pastoureau, R. Gandilhon, J.-L. Chassel, G. Cahen, M. de Framond). Longtemps sous-exploité, le sceau est apparu ces dernières années comme un document d'une richesse multiforme, aussi bien dans le domaine juridique, institutionnel et diplomatique que dans le domaine archéologique et iconographique ${ }^{57}$. Désormais, tous les médiévistes sem- 
blent connaître cette richesse documentaire exceptionnelle - ce qui n'était sans doute pas vrai il y a vingt ans - et, malgré les difficultés matérielles que pose sa conservation, le sceau devrait constituer dans les années à venir un des documents " carrefour » des études médiévales. Après la miniature (sur laquelle il présente l'avantage d'une datation beaucoup plus précise), le sceau représente la source d'images quantitativement la plus importante que le Moyen Age nous ait laissée.

Grâce aux Archives de France, la politique de recensement et de publication des sceaux médiévaux, longtemps interrompue, a été relancée, soit sous forme d'inventaires, soit sous forme de corpus $^{58}$. Souhaitons que ce que les Archives nationales et départementales ont entrepris en ce domaine soit poursuivi à grande échelle et imité par les grandes bibliothèques; notamment par la Bibliothèque nationale qui abrite en son sein plusieurs dizaines de milliers d'empreintes de sceaux médiévaux, la plupart inconnues de tous les répertoires.

\section{Onomastique}

L'onomastique (étude des noms de lieux : toponymie, et des noms de personnes : anthroponymie) continue de rencontrer une certaine réserve dans les milieux universitaires, ceux-ci la considérant volontiers comme dépourvue de méthode scientifique. De fait, trop de travaux accomplis par des amateurs sont livrés au grand public, toujours avide d'étymologies. Or les matériaux que traite l'onomasticien sont des mots : ils exigent donc de sa part une double formation, celle d'un linguiste et d'un philologue, mais aussi, parce qu'ils sont les témoignages d'un passé révolu, celle d'un historien. Formation spécifique, qu'il n'est pas facile d'acquérir : dans une enquête menée il y a quelques années ${ }^{59}$, $P$. Fabre a constaté une indifférence quasi générale, de la part des universités françaises, envers cette discipline.

Celle-ci cependant a ses spécialistes, qui se réunissent en congrès internationaux tous les trois ans $\left(16^{\mathrm{e}}\right.$ congrès à Québec, 1987). En France, la Société française d'onomastique, peu 
nombreuse mais active, organise des colloques régionaux depuis 1978. La Revue internationale d'onomastique, fondée par A. Dauzat, disparue après 1977 au décès de l'éditeur, a été remplacée en 1983 par une Nouvelle Revue d'onomastique dont personne ne met en doute la qualité scientifique. A Dijon a été créée, voici quelques années, par G. Taverdet, professeur à l'université de Dijon, une Association bourguignonne de dialectologie et d'onomastique (ABDO) qui diffuse des monographies à caractère pédagogique, succinctes, mais de bon niveau.

C'est dire qu'un peu partout du travail sérieux s'accomplit, qu'il faut savoir repérer dans l'abondance des publications ${ }^{60}$. En voici un aperçu :

- Les manuels : les ouvrages traditionnels d'A. Vincent et d'A. Dauzat ${ }^{61}$ n'ont pas encore été remplacés. A signaler cependant un utile manuel élaboré par deux professeurs de l'université de Montpellier, manuel qui présente conjointement, de façon claire et pédagogique, états de questions, acquis et problèmes de la toponymie et de l'anthroponymie française ${ }^{62}$.

- Les dictionnaires topographiques départementaux : dans la collection officielle, celui de la Seine-Maritime; hors collection et de moindre valeur, utiles néanmoins, celui de l'Ardèche (P. Charrié) et celui de l'Oise (E. Lambert); publications analogues, avec formes anciennes, pour la Manche (F. de Beaurepaire) et la Haute-Vienne (M. Villoutreix); en refonte, avec préoccupations étymologiques, ceux de l'Hérault (F.-R. Hamlin) et de la Moselle ( $\mathrm{H}$. Hiégel).

- Pour l'ensemble du territoire, le Dictionnaire étymologique des noms de rivières et de montagnes en France commencé par A. Dauzat, poursuivi par G. Deslandes, publié enfin par $\mathrm{Ch}$. Rostaing, rendra des services, mais plutôt comme orientation de recherches que comme solutions définitives aux problèmes de l'hydronymie et de l'oronymie.

- Les corpus : avec ses trois volumes de Noms de personne sur le territoire de la Gaule du $V^{e}$ au XII siècle, M.-Th. Morlet a fourni une très importante contribution à la connaissance de l'anthroponymie haut-médiévale. L'ouvrage est fondé sur une somme considérable de sources imprimées.

- Les textes : tous documents de l'histoire présentant évi- 
demment quelque intérêt onomastique : pouillés, cartulaires et censiers, livres d'estimes, etc. Il n'y a pas lieu d'y insister ici.

- Le travail universitaire : ont été publiées quelques thèses d'Etat, en toponymie, en hydronymie, en anthroponymie ${ }^{63}$.

- La recherche collective, notamment en anthroponymie : on doit à l'équipe de L. Pérouas une étude tout à faire neuve sur les prénoms en Limousin depuis un millénaire. Rendant compte de cette publication, le regrettté $J$. Herbillon écrivait naguère : "L'étude des prénoms, secteur de l'anthroponymie, a d'abord été du domaine des linguistes qui s'efforçaient d'expliquer philologiquement les formes recueillies. L'évolution des méthodes historiques et les poussées conjointes des recherches démographiques et anthropologiques à partir des années cinquante n'ont pas tardé à convaincre les historiens de l'importance de l'étude systématique des prénoms. Cette importance avait déjà été soulignée par M. Bloch ${ }^{64}$. »

On sait que, nom unique jusqu'à l'orée du deuxième millénaire, le prénom s'est ensuite complété d'un surnom individuel, ancêtre de notre "nom de famille ". A l'université de Tours, un groupe de chercheurs, autour de M. Bourin, a entrepris d'examiner plus précisément où, quand et comment on est passé de ce nom unique au système à deux éléments. La démarche est celle d'historiens attentifs à la nature et à la valeur des sources utilisées, aussi bien qu'aux catégories sociales considérées. Les premiers résultats de l'enquête, parus dans un récent volume ${ }^{65}$, sont encore fragmentaires; mais une méthode de recherche est ainsi élaborée, qui vise à décrire statistiquement les phénomènes observés au lieu d'accumuler les anecdotes. Elle devrait être féconde.

En toponymie, la grande vogue des substrats préindo-européens est passée. L'utilisation des techniques informatiques, qui permettent maintenant de gérer d'énormes masses d'informations, a ramené les chercheurs vers la réalité que représentent les toponymes. A travers la microtoponymie, presque inexplorée jusqu'ici, il va être possible de détecter la survivance et d'étudier la répartition de nombre d'appellatifs, certains d'entre eux donnant, par exemple, de précieuses indications sur l'occupation du sol ${ }^{66}$. La dialectologie prend ici un rôle majeur. 
En anthroponymie, l'essor de la recherche généalogique apporte de nouvelles informations sur l'histoire des noms de famille. Mais l'anthroponymie médiévale aurait besoin d'avoir à disposition plus de matériaux datés et localisés, tels ceux qu'a publiés R. Berger ${ }^{67}$. Une familiarité avec le nom médiéval et des traitements « à la main » restent, et pour longtemps encore, indispensables avant tout recours aux techniques informatiques.

Certes, aucun historien ne peut contester l'utilité de l'onomastique bien pratiquée. Le vrai problème, qui est celui de beaucoup d'autres sciences auxiliaires, est celui de la formation de l'onomasticien et les débouchés qui lui sont offerts.

Le tableau qui précède suffit à montrer que les sciences dites auxiliaires ne sont plus, ou plus seulement, des auxiliaires ; mais pas davantage des sciences fondamentales, au sens des Grundwissenschaften d'outre-Rhin, en ce qu'elles ne se nourrissent pas de leur propre mouvement, mais visent toujours et immédiatement à servir l'histoire. Si les acquis ont été détaillés, il reste à évoquer les zones d'ombre qui demeurent. En premier lieu, les problèmes d'une édition qui est loin de suivre le mouvement et où, même généralisées, les nouvelles techniques informatiques ne résoudront pas tout: exiguité du public, problèmes de financement, coût de reproduction des sources figurées. Et si, ici comme ailleurs, les congrès se multiplient, le problème reste entier d'une base trop étroite dans l'enseignement supérieur : l'absence de réédition du Manuel de R. Delort, précisément publié en 1969, est particulièrement symptomatique.

En résumé, les progrès des sciences auxiliaires en France dans les vingt dernières années ont associé vitalité et inachèvement, foisonnement et dispersion, voire éclatement et confidentialité. En bien des domaines, le mouvement doit encore faire ses preuves. Le temps des synthèses est loin d'être arrivé et, même si nous sommes parvenus à livrer un instantané point trop flou, il est fort peu probable que le cadre chronologique, 1969-1989, demeure longtemps bien pertinent. 


\section{Notes}

1. R. Delort, Introduction aux sciences auxiliaires de l'histoire, Paris, Colin, 1969, p. 10.

2. B. Garnier, J.-C. Hocquet et D. Woronoff, Introduction à la métrologie historique, Paris, Economica, 1989.

3. Les Sources de l'histoire économique et sociale du Moyen Age, sous la direction de R.-H. Bautier, Paris, CNRS, 1968- (3 vol. pour le Sud-Est ; 1 vol. paru pour les états de la maison de Bourgogne; domaine royal en préparation). $Y$ joindre les articles de R. Favreau sur les sources du Poitou, de M. Nortier sur celles de la Normandie, de G. Peyronnet sur les sources anglaises concernant la Bretagne.

4. Il convient d'ajouter que l'Etat des inventaires des Archives nationales (Paris, Archives nationales, diff. La Documentation française, 1985-, 2 vol. parus) signale systématiquement les éditions de documents. Les états publiés peuvent être mis à jour au moyen des Rapports annuels multigraphiés de la Direction des Archives de France et, pour les seuls instruments de recherche imprimés, grâce à la bibliographie annuelle publiée dans la Gazette des Archives. Catalogues d'expositions et dossiers de services éducatifs sont des mines de renseignements, trop souvent méconnues (ceux des Archives nationales ont été recensés dans l'Etat des inventaires, op. cit., t. 4, p. 226-237 ; pour les Archives départementales, cf. la publication de l'Association des archivistes français, Par les archives, apprendre l'histoire: trente ans d'activité des services éducatifs des Archives de France, bibliographie des travaux..., Paris, 1982).

5. Diplomatica et Sigillographica... Normalisation internationale des éditions de documents et... vocabulaire international de la diplomatique et de la sigillographie, Saragosse (Folia Caesaraugustana, 1) [1984], actuellement en cours de refonte pour publication définitive. Une édition partielle et préparatoire concernant les normes de publication a paru dans Bulletin philologique et historique, 1976, p. 9-54.

6. A titre d'exemples : M. Zimmermann, «Protocoles et préambules dans les documents catalans du $\mathrm{X}^{c}$ au XII ${ }^{c}$ siècle... ", Mélanges de la Casa de Velázquez, t. 10, 1974 , p. 41-76, et t. 11, 1975, p. 51-79. M. Rouche, "Les survivances antiques dans trois cartulaires du Sud-Ouest de la France », Cahiers de civilisation médiévale, t. 23, 1980, p. 93-108.

7. J. Dufour, « Etat et comparaison des actes faux ou falsifićs intitulés au nom des Carolingiens trançais (840-987) ", in Fälschungen im Mittelalter, Hanovre, Hahn, 1988 , t. 4 , p. $167-210$.

8. Pour un bilan d'ensemble, cf. R.-H. Bautier, " Les sources documentaires de l'histoire de France au Moyen Age ", in Tendances, Perspectives et Méthodes de l'histoire médiévale (100 congrès national des Sociétés savantes, Paris, 1977), Paris, CTHS, 1977, p. 215-248. - , * Les orientations de la diplomatique en Europe depuis la fin de la Seconde Guerre mondiale », in Cento anni di cammino, Vatican, Città del 


\section{L'HISTOIRE MÉDIÉVALE EN FRANCE}

Vaticano, 1986, p. 101-145 (dont la bibliographie devrait paraitre à part). Il serait injuste de ne pas évoquer, parmi les collègues étrangers qui ont contribué à l'édition et à l'étude des sources françaises ou concernant la France, seules ici prises en compte, les regrettés J. Benton et W.M. Newman, ainsi que H. Atsma, J. Baldwin, E.A.R. Brown, J.-P. Devroey, M. Jones, R.A. Lodge, D. Lohrmann, R. Mantou, J.H. Pryor, J. Pycke, P. Ruelle, B.-M. Tock, K.-F. Werner, entre autres.

9. R.-H. Bautier, "L'authentification des actes privés dans la France médiévale », sous presse. M. Parisse, "Remarques sur les chirographes et les chartes-parties antérieurs à 1120 », Archiv für Diplomatik, t. 32, 1986, p. 546-567.

10. Collaboration française au lancement de la Gallia Pontificia. B. Barbiche, Les Actes pontificaux originaux des Archives nationales (1198-1415), Vatican, Città del Vaticano, 1975-1982, 3 vol.

11. On pourra y ajouter, entre autres, P. Gérard, "Les sources de l'histoire oénédictine conservées dans les archives du Grand Sud-Ouest ", Cahiers de Fanjeaux, $\mathrm{n}^{\circ}$ 19, Toulouse, 1984, p. 23-51.

12. A signaler, modèle du genre, l'étude d'H. Flammarion, "Une équipe de scribes au travail au XIII ${ }^{e}$ siècle : le grand cartulaire du chapitre cathédral de Langres ", Archiv für Diplomatik, t. 28, 1982, p. 271-305.

13. Pour un panorama chronologiquement plus vaste, mais restreint à la paléographie, cf. D. Muzerelle, "Un siècle de paléographie latine en France », in Un secolo di paleografia e diplomatica (1887-1986), per il centenario dell'Istituto di paleografia dell'Università di Roma, Rome, Gela, 1988, p. 130-158.

14. Ductus, ou la Formation de l'alphabet moderne, Paris, Les Films verts, 1976. Cf. E. Poulle, "Une histoire de l'écriture », Bibliothèque de l'Ecole des chartes, t. 135,1977 , p. $137-144$.

15. J. Mallon, De l'écriture. Recueil d'articles publiés de 1937 à 1981, Paris, CNRS, 1982.

16. Chartae latinae antiquiores : Facsimile-Edition of the Latin Charters prior to the Ninth Century, A. Bruckner et R. Marichal éd., Dietikon-Zurich, Urs Graf Verlag, Part XIII-XIX, France I-VII, publié par H. Atsma, J. Vezin et al., 1981-1987.

17. P. Gasnault et J. Vezin, Documents comptables de Saint-Martin de Tours à l'époque mérovingienne, publiés... avec une étude paléographique, Paris, Bibliothèque nationale, 1975 .

18. F. Gasparri, L'Ecriture des actes de Louis VI, Louis VII et Philippe Auguste, Genève, Droz, Paris, Minard, 1973.

19. Par exemple, M. Parisse, "Un scribe champenois et l'évolution de son écriture ", Archiv für Diplomatik, t. 29, 1983, p. 229-241. O. Merisalo, La Langue et les Scribes: études sur les documents en langue vulgaire de La Rochelle, Loudun, Châtellerault et Mirebeau au XIII' siècle, Helsinki, Societas scientiarum Fennica, 1988.

20. E. Poulle, Paleographie des écritures cursives en France, $d u X V^{e}$ au XVII siècle : recueil de fac-similés de documents parisiens..., Genève, Droz, 1966.

21. H. Millet et E. Poulle, Le Vote de la soustraction d'obédience en 1398, t. 1, Introduction. Edition des bulletins de vote. Fac-similé des bulletins de vote, Paris, CNRS, 1988.

22. A signaler tout particulièrement : Testaments provençaux du Moyen Age: documents paléographiques, sous la direction d'A. Ramière de Fortanier, Marseille, Archives de la ville de Marseille et Institut historique de Provence, 1979.

23. Catalogue des manuscrits en écriture latine portant une indication de date, de lieu ou de copiste, sous la direction de Ch. Samaran et R. Marichal, Paris, CNRS, t. 1, 


\section{SCIENCES AUXILIAIRES}

Musée de Condé et Bibliothèques parisiennes, 1959 ; t. 2-4 (1), Bibliothèque nationale, Fonds latin, Nouvelles Acquisitions latines et Petits Fonds divers, 1962-1981 ; t. 5, Est de la France, 1965 ; t. 6, Bourgogne, Centre, Sud-Est et Sud-Ouest de la France, 1968 ; t. 7, Ouest de la France et Pays de Loire, 1984.

24. J. Vezin, Les « Scriptoria » d'Angers au XI siècle, Paris, Champion, 1974.

25. J. Dufour, La Bibliothèque et le "Scriptorium " de Moissac, Genève-Paris, Droz, 1972. M.-C. Garand, "Copistes de Cluny au temps de saint Maïeul (948994) ", Bibliothèque de l'Ecole des chartes, t. 136, 1978, p. 5-36.

26. M.-C. Garand, "Auteurs latins et autographes des $\mathrm{XI}^{\mathrm{e}}$ et $\mathrm{XII}^{\mathrm{e}}$ siècles ", Scrittura e Civiltà, t. 5, 1981, p. 77-104.

27. D. Muzerelle, Vocabulaire codicologique : répertoire méthodique des termes français relatifs aux manuscrits, Paris, CEMI, 1985.

28. Les Techniques de laboratoire dans l'étude du manuscrit (actes du colloque international du CNRS, Paris, 13-15 septembre 1972), Paris, CNRS, 1974.

29. M. Zerdoun Bat-Yehouda, Les Encres noires au Moyen Age (jusqu'à 1610), Paris, CNRS, 1983.

30. M. Zerdoun Bat-Yehouda, Les Papiers filigranés médiévaux: essai de méthodologie, Turnhout, Brepols, 1989, 2 vol.

31. Histoire des bibliothèques françaises, Paris, Promodis, 1988-1989, 2 vol. ; t. 1, Les Bibliothèques médiévales, du ve siècle à 1530 , sous la direction d'A. Vernet ; t. 2, Les Bibliothèques sous l'Ancien Régime, sous la direction de C. Jolly.

32. A.-M. Genevois, J.-F. Genest et A. Chalandon, Bibliothèques de manuscrits médiévaux en France : relevé des inventaires, du vII au XVIII siècle, Paris, CNRS, 1987.

33. A. Vernet, La Bibliothèque de l'abbaye de Clairvaux, du XII au XVIII' siècle,

t. 1, Catalogues et Répertoires, Paris, CNRS, 1980.

34. G. Ouy et V. Gerz-Von Büren, Le Catalogue de la bibliothèque de l'abbaye de Saint-Victor de Paris, de Claude de Grandrue (1514), Paris, CNRS, 1983.

35. D. Nebbiai-Dalla Guarda, La Bibliothèque de l'abbaye de Saint-Denis en France, du IX $X^{e}$ au XVIII siècle, Paris, CNRS, 1985.

36. D. Willmann, Bibliothèques ecclésiastiques au temps de la papauté d'Avignon, Paris, CNRS, 1980.

37. C. Bozzolo et E. Ornato, "Pour une codicologie 'expérimentale' ", Scrittura e Civiltà, t. 6, 1982, p. 263-302. C. Bozzolo, D. Coq, E. Ornato et D. Muzerelle, «Une machine au fonctionnement complexe : le livre médiéval », in La Présentation du texte, Paris, 1989.

38. C. Bozzolo et E. Ornato, Pour une histoire du livre manuscrit au Moyen Age. Trois essais de codicologie quantitative, $2^{c}$ éd. avec supplément, Paris, CNRS, 1983.

39. C. Bozzolo, D. Coq, E. Ornato et D. Muzerelle, « Noir et blanc : premiers résultats d'une enquête sur la mise en pages dans le livre médiéval ", in Il Libro e il Testo (convegno internazionale, Urbino, 1983), Urbino, Quattroventi, 1984, p. 195222. - « Page savante, page vulgaire : étude comparative de la mise en pages des livres écrits ou imprimés en France au XV siècle ", in La Présentation du livre (colloque de Paris-X-Nanterre, 1985), Nanterre, Centre de recherches du département de français de Paris-X-Nanterre, 1987, p. 121-133.

40. G. Nahon, "L'épigraphie ", in Art et Archéologie des Juifs en France médiévale, sous la direction de B. Blumenkranz, Toulouse, Privat, 1980, p. 95-132.

41. R. Favreau, Les Inscriptions médiévales, Turnhout, Brepols (« Typologie des sources du Moyen Age occidental », 35), 1979. 


\section{L'HISTOIRE MÉDIÉVALE EN FRANCE}

42. R. Favreau, «L'épigraphie médiévale », Cahiers de civilisation médiévale, $\mathrm{t}$. 12, 1969, p. 393-398. —, " L'épigraphie, reflet d'une culture et d'une foi » (congrès de Graz, 1988) (à paraître). - " "L'épigraphie médiévale : naissance et développement d'une discipline »Comptes rendus de l'Académie des inscriptions et belleslettres, 1989. - " "Fonctions des inscriptions médiévales ", Cahiers de civilisation médiévale, t. 32, 1989, p. 203-232.

43. L. Pressouyre, * Lecture d'une inscription du XIr siècle à Saint-AntoninNoble-Val, Tarn-et-Garonne ", Bulletin de la Société nationale des antiquaires de France, 1986, p. 255-268. R. Favreau, « L'inscription du tympan nord de San Miguel d'Estella », Bibliothèque de l'Ecole des chartes, t. 123, 1975, p. 237-246. —, étude à paraître sur Colius Sedulius.

44. J. Duplessy, Les Monnaies françaises royales de Hugues Capet a Louis XVI (987-1793), t. 1, Hugues Capet-Louis XII, Paris, Platt, 1988.

45. La Commission internationale de numismatique publie, à l'occasion de chaque congrès international de numismatique, une bibliographie critique des travaux récents : A Survey of Numismatic Research (1966-1971), New York, International Numismatic Commission, 1973. - 1972-1977, Berne, International Numismatic Commission, 1979. - 1978-1984, Londres, International $\Lambda$ ssociation of Professional Numismatics Special Publication, $\mathrm{n}^{\circ}$ 9, 1986.

46. J. Lafaurie, « Le gros tournois en France », Numismaticky Sbornik, t. 12, 1970-1971, p. 49-64.

47. J. Lafaurie, « Numismatique : des Carolingiens aux Capétiens », Cahiers de civilisation médiévale, t. 13, 1970, p. 117-137, pl. I-IV.

48. J. Duplessy, Les Trésors médiévaux et modernes découverts en France, t. 1, 751-1223, Paris, Bibliothèque nationale, 1985.

49. F. Dumas, « Les monnaies de Raoul, roi de France (923-936) », in Mélanges de numismatique, d'archéologie et d'histoire offerts à Jean Lafaurie, Paris, Société française de numismatique, 1980, p. 212-222, pl. XI-XXII, à compléter par un article du même auteur dans la Revue numismatique de 1981 et diverses communications de J. Duplessy dans le Bulletin de la Société française de numismatique.

50. F. Dumas-Dubourg, « La monnaie dans le royaume au temps de Philippe Auguste ", in La France de Philippe Auguste (actes du colloque 602 du CNRS, Paris, 29 sept. -4 oct. 1980), Paris, CNRS, 1983, p. 543-574. F. Dumas et J.-N. Barrandon, Le Titre et le Poids de fin des monnaies sous le règne de Philippe Auguste, ParisValbonne, CNAS (Cahiers Ernest-Babelon, t. 1), 1982.

51. F. Dumas, "Les monnaies normandes $\left(\mathrm{X}^{\mathrm{e}}-\mathrm{XII}{ }^{\mathrm{e}}\right.$ siècle $)$, avec un répertoire de trouvailles ", Revue numismatique, $6^{\mathrm{e}}$ série, t. $21,1979, \mathrm{p} .84-140$, pl. XV-XXI, à compléter par diverses communications du même auteur et de J. Pilet-Lemière dans le Bulletin de la Société française de numismatique.

52. L. Musset et al., Aspects de la société et de l'économie dans la Normandie médiévale ( $X^{e}-X I I r^{e}$ siècle), Caen, Annales de Normandie (Cahiers des Annales de Normandie, t. 22), 1988, en particulier p. 65-89.

53. F. Dumas-Dubourg, Le Monnayage des ducs de Bourgogne, Louvain-laNeuve, Séminaire de numismatique Marcel-Hoc, 1988.

54. M. Bompaire, "La monnaie de Pont-de-Sorgues dans la première moitié du XIV siècle ", Revue numismatique, $6^{\mathrm{e}}$ série, t. 25, 1983, p. 139-176, et * La monnaie comtale de Blois au début du XIve siècle ", Bulletin de la Société française de numismatique, $39^{e}$ année, 1984, p. 460-464. Pierre Capra, * Les espèces, les ateliers, les frappes et les émissions monétaires en Guyenne anglo-saxonne aux XIV et $\mathbf{X V}^{e}$ 


\section{SCIENCES AUXILIAIRES}

siècles ", Numismatic Chronicle, CXXXIX a., 1979, p. 139-154, et CXL a., 1980, p. 132-164. V. Chomel, « Le dauphin Humbert II et l'atelier monétaire de Visan (1338-1345) ", Provence historique, t. 23, 1973, p. 139-145.

55. M. Bompaire, « Un livre de changeur languedocien du milieu du XIV siècle ", Revue numismatique, $6^{\mathrm{e}}$ série, t. 29, 1987, p. 118-183.

56. M. Pastoureau, Traité d'héraldique, Paris, Picard, 1979.

57. R. Gandilhon et M. Pastoureau, Bibliographie de la sigillographie française, Paris, Picard, 1982. M. Pastoureau, Les Sceaux, Turnhout, Brepols (« Typologie des sources du Moyen Age occidental ", 36), 1983. A titre d'exemple R.-H. Bautier, « Echanges d'influences dans les chancelleries souveraines du Moyen Age d'après les types de sceaux de majesté ", Comptes rendus de l'Académie des inscriptions et belleslettres, 1968, p. 192-220.

58. Archives nationales, Corpus des sceaux français du Moyen Age, t. 1, Les Sceaux des villes, par B. Bedos, Paris, Archives nationales, 1980 ; t. 2, Les Sceaux des rois de France, par M. Dalas-Garrigues (sous presse).

59. P. Fabre, « Enquête sur l'enseignement et la recherche en onomastique dans les universités françaises , in L'Onomastique témoin des langues disparues (actes du colloque d'onomastique romane de Dijon), Dijon, ADBO, 1982, p. 1-14.

60. Près de 8000 références sont recensées par M. Mulon, L'Onomastique française: bibliographie des travaux publiés de 1960 a 1985, Paris, Archives nationales, 1987.

61. A. Vincent, Toponymie de la France, Bruxelles, Librairie générale, 1937. A. Dauzat, La Toponymie française, éd. revue, Paris, Payot, 1960. -, Traité d'anthroponymie française : les noms de famille de France, $3^{c}$ éd. revue et complétée par M.-Th. Morlet, Paris, Guénégaud, 1977.

62. Ch. Baylon et P. Fabre, Les Noms de lieux et de personnes, Paris, Nathan, 1982.

63. J.-M. Ricolfis, Les Noms de lieux du pays niçois, Lille, université Lille-III, Paris, Champion, 1980. P. Gauthier, Toponymie de la côte vendéenne, Lille, université Lille-III, 1977. P. Fabre, L'Affluence hydronymique de la rive droite du Rhône, Montpellier, Centre d'études occitanes, 1980. A. Compan, Les Noms de personnes dans le comté de Nice aux XIIr, XIV et XV siècles, Lille, université LilleIII, Paris, Champion, 1976.

64. J. Herbillon, compte rendu de L. Pérouas, Léonard, Marie, Jean et les Autres (Paris, CNRS, 1984), in Revue belge de philologie et d'histoire, t. 66, 1988, p. 910.

65. Genèse médiévale de l'anthroponymie moderne, Tours, université FrançoisRabelais, 1989.

66. Cf. les nombreux travaux de Ch. Higounet et les actes des VIII Journées internationales d'histoire (Flaran, 1986) : Toponymie et défrichements, Auch, Commission d'histoire de Flaran, 1988.

67. Notamment Nécrologe de la Confrérie des jongleurs et bourgeois d'Arras (11941361), Arras, Commission départementale des Monuments historiqucs, 1963-1970, 2 vol. 